IMAFF-FM-02/14

hep-th/0212348

\title{
Some Issues in Conformal Field Theory with Boundaries and Crosscaps
}

\author{
Beatriz Gato-Rivera \\ Instituto de Matemáticas y Física Fundamental, CSIC, \\ Serrano 123, Madrid 28006, Spain* \\ NIKHEF-H, Kruislaan 409, NL-1098 SJ Amsterdam, The Netherlands
}

\begin{abstract}
This is a brief introduction to the subject of Conformal Field Theory on surfaces with boundaries and crosscaps, which describes the perturbative expansion of open string theory.
\end{abstract}

December 2002

\footnotetext{
*Invited talk given at the Conference 'Conformal Field Theory and Integrable Systems', held by Landau Institute, Russia, September 15-21, 2002. e-mail address: bgator@imaff.cfmac.csic.es
} 
I am going to give a brief introduction to the subject of CFT on surfaces with boundaries and crosscaps, which is the CFT description of perturbative open string theory. Let me start with a small historical remark. During many years open string theory has been much less popular than closed string theory and there were reasons for it. On the one hand, it was believed that open strings had nothing to do with the real world since they could not provide realistic models for particle Physics, in contrast to the case of the heterotic strings. On the other hand, open strings are much more difficult to deal technically than closed strings. For these reasons it is understandable that, from the 'rise' of String Theory in 1985 until 1995, the bulk of string theoretists concentrated on closed strings, mainly heterotic strings, while only very few paid attention to open strings [1] [7]. This situation changed drastically when Polchinski discovered D-branes in 1995 [2]. The reason is that, apart from the genuine interest on these new objects to which the open strings attach, it was also shown that the open strings are dual to closed strings, in particular to the heterotic strings, and finally it appeared that all known string theories are related to each other (as a matter of fact, as everybody knows by now, it seems that they are different limits of one and the same theory in 11 dimensions, known as M-theory). This was the end of the 'discrimination' against open string theory which at present is a very active field. However, the CFT treatment of open strings is been worked out by very few groups and this is the reason I decided to give an introductory talk in this conference.

In what follows I will describe the basic ingredients necessary for the study of CFT's on surfaces with boundaries and crosscaps. Let us first review the 2-dimensional surfaces involved in the perturbative expansion of string theories at one-loop, that is the worldsheets swept by the strings when moving in space-time. In string theory at one-loop one finds four different types of topologically inequivalent worldsheets: the torus, the Klein bottle, the annulus, and the Möbius strip. Depending on the type of string theory, some of these surfaces may or may not appear. For closed oriented strings only the torus appear whereas for closed unoriented strings one has also the Klein bottle. For open oriented strings one finds the annulus plus the torus corresponding to the closed sector of the theory and, finally, for open unoriented strings the four types of worldsheets appear. The 'direct channel' surfaces (with the proper time making a loop) contain the information about the complete spectrum of the CFT defined on them; that is, the amplitudes of these surfaces represent the partition functions of the theory that give the number of states level by level. But one can also look at these surfaces from the 'transverse channel' point of view, that is exchanging the space and time coordinates of the worldsheet. The torus does not change, but the annulus, the Möbius strip and the Klein bottle look quite different. The annulus is converted into a cylinder between two boundaries, that shows the propagation of closed strings between the two boundaries, the Möbius strip is converted into a cylinder between a boundary and a crosscap (the Möbius strip has only one boundary), and the Klein bottle turns into a cylinder between two crosscaps (a 
crosscap is a boundary with the opposite sides identified).

Now let us consider any 2-dimensional surface with boundaries and crosscaps. Let us call the closed string chiral algebra as $G_{L} \otimes G_{R}$, with generators $W_{n}$ and $\hat{W}_{n}$. At a boundary or crosscap only the diagonal combination survives because these 'defects' interchange left and right-movers. As a result, the boundary states $|B\rangle$ and the crosscap states $|C\rangle$ must satisfy the conditions

$$
\left[W_{n}-(-1)^{h_{w}} w \hat{W}_{-n}\right]|B\rangle=0, \quad\left[W_{n}-(-1)^{h_{w}+n} w \hat{W}_{-n}\right]|C\rangle=0
$$

where $h_{w}$ is the conformal weight of the generator $W_{n}$ and if $w \neq 1$ this is called a symmetry breaking boundary condition [3][4]. There is a formal solution to these conditions, given by the so-called Ishibashi states [5]

$$
|i\rangle_{B}=\sum_{l}|i, l\rangle_{L} \otimes U_{B}|i, l\rangle_{R}, \quad|i\rangle_{C}=\sum_{l}|i, l\rangle_{L} \otimes U_{C}|i, l\rangle_{R}
$$

where $i$ denotes the ground state, $l$ the level, and $U_{B}$ and $U_{C}$ are anti-unitary operators. The physical boundary states and crosscap states are linear combinations of the Ishibashi states

$$
\left|B_{a}\right\rangle=\sum_{i} B_{a i}|i\rangle_{B}, \quad|C\rangle=\sum_{i} \Gamma_{i}|i\rangle_{C}
$$

where $B_{a i}$ and $\Gamma_{i}$ are called boundary and crosscap coefficients, respectively. The label $a$ indicates that a CFT can have different sets of boundaries, whereas it can have only one type of crosscap.

The boundary and crosscap coefficients are very important quantities in open string theory since they contain information about the spectrum of the string states as well as information about the D-branes and orientifold planes (O-planes for short). These coefficients are constrained by sewing constraints, which are rather difficult to solve, and also by integrality and positivity conditions that are very restrictive but much easier to solve than the sewing constraints. The origin of the integrality and positivity conditions is the fact that the partition functions, which give the state multiplicities, are given by the one-loop amplitudes and, in the transverse channel, these amplitudes depend explicitely on the boundary and crosscap coefficients. For example, from the annulus, where open strings are running in the direct channel, one gets the integrand

$$
N^{a} N^{b} A_{a b}^{i} \chi^{i}(\tau / 2)
$$

where $N^{a}, N^{b}$ are the Chan-Paton factors at the ends of the strings, $\chi^{i}$ is the character 
of the representation $i$, and $A_{a b}^{i}$ can be obtained from the transverse channel, having the expression

$$
A_{a b}^{i}=\sum_{j} S_{j}^{i} B_{j a} B_{j b}
$$

where $S$ is the modular matrix, $S: \tau \rightarrow-1 / \tau$. Now from the direct channel one gets that

$$
A_{a b}^{i} \in \mathbf{Z} \geq 0
$$

In the same manner, from the Möbius strip and the Klein bottle one obtains the conditions

$$
1 / 2\left(A_{a a}^{i}+M_{a}^{i}\right) \in \mathbf{Z} \geq 0, \quad 1 / 2\left(Z_{i i}+K^{i}\right) \in \mathbf{Z} \geq 0
$$

with

$$
M_{a}^{i}=\sum_{j} P_{j}^{i} B_{j a} \Gamma_{j} \in \mathbf{Z}, \quad K^{i}=\sum_{j} S_{j}^{i} \Gamma_{j} \Gamma_{j} \in \mathbf{Z}
$$

where the $\mathrm{P}$ matrix is defined as [7][8] $P=\sqrt{T} S T^{2} S \sqrt{T}, T$ being the modular matrix, $T: \tau \rightarrow \tau+1$, and $Z_{i i}$ comes from the torus partition function. (Let us remind that the torus partition function can be expressed as $\left.\sum_{i j} \chi_{i}(\tau) Z_{i j} \chi_{j}(\tau)\right)$.

Another important consistency condition is the tadpole cancellation. It happens that an open unoriented string with arbitrary Chan-Paton factors is in general inconsistent due to infrared divergences in one-loop amplitudes. This is very easy to see in the transverse channel where, by factorization, the 'cylinder' decomposes as the product of the propagator times the tadpoles corresponding to the extremes (boundary tadpoles and/or crosscap tadpoles). Therefore the tadpoles must cancel, what implies that the Chan-Paton factors must be adjusted to some specific values. But the Chan-Paton factors reflect the gauge group of the theory and therefore the tadpole cancellation fixes the possible allowed gauge groups.

Now let us see some examples of solutions for the boundary and crosscap coefficients. For any CFT such that the modular invariant is the charge conjugation, that is $Z_{i j}=C_{i j}$, Cardy found the boundary coefficients [6]

$$
B_{a i}=\frac{S_{a i}}{\sqrt{S_{0 i}}}
$$

and the 'Rome group' (Sagnotti and collaborators) found the crosscap coefficients [8][9] 


$$
\Gamma_{i}=\frac{P_{0 i}}{\sqrt{S_{0 i}}}
$$

These results have been generalized first of all by allowing more general simple current generated Klein bottles, and secondly to arbitrary simple current modular invariants, as classified in refs. [10] and [11]. This generalization was pioneered by the Rome group, who worked out the first examples for $\mathrm{SU}(2)$ [8], and was completed in a series of papers by Fuchs and Schweigert [12] and by a group from Amsterdam (Huiszoon, Schellekens and Sousa) [13]. The final result can be summarized very concisely in a single formula for the boundary and crosscap coefficients [14]. The simplest example, which corresponds to a non-standard Klein bottle choice specified by a simple current $k$, reads

$$
B_{a i}^{k}=\frac{S_{a i}}{\sqrt{S_{k i}}}, \quad \Gamma_{i}^{k}=\frac{P_{k i}}{\sqrt{S_{k i}}}
$$

where the index $k$ refers to the Klein bottle simple current.

To finish let me say a few words about the meaning of boundaries and crosscaps in space-time. The boundaries are glued to D-branes as it is easy to see intuitively in the open string picture where the ends of the strings are attached to the D-brane. Crosscaps, however, are very difficult to visualize since, first of all, they are not even localized. This means that while one can 'see' a boundary, even touch it with the fingers on an annulus or a Möbius strip, one cannot do the same with a crosscap. In space-time crosscaps are related to orientifold configurations in such a way that a single crosscap can correspond to a sum of orientifolds, not to just one. (Orientifolds are static hyperplanes where left and right-movers are identified, they are like mirrors for charge conjugation of D-branes).

\section{Acknowledgements}

I am very grateful to A. Sagnotti and A.N. Schellekens for getting me started on the subject of CFT with boundaries and crosscaps.

\section{References}

[1] C.P. Burgess and T.R. Morris, Nucl. Phys. B291 (1987) 256 ; Nucl. Phys. B291 (1987) 285

M.R. Douglas and B. Grinstein, Phys. Lett. B183 (1987) 52

N. Marcus and A.Sagnotti, Phys. Lett. B188 (1987) 58

J.A. Harvey and J.A. Minahan, Phys. Lett. B188 (1987) 44 
G. Pradisi and A. Sagnotti, Phys. Lett. B216 (1989) 59

M. Bianchi and A. Sagnotti, Phys. Lett. B231 (1989) 389

J. Govaerts, Phys. Lett. B220 (1989) 77

P. Horava, Phys. Lett. B231 (1989) 251.

[2] J. Polchinski, Phys. Rev. Lett. 75 (1995) 4724

[3] J. Fuchs and Ch. Schweigert, Nucl. Phys. B530 (1998) 99

[4] A. Recknagel and V. Schomerus, Nucl. Phys. B531 (1998) 185

[5] N. Ishibashi, Mod. Phys. Lett. A4 (1989) 251

[6] J. Cardy, Nucl. Phys. B324 (1989) 581

[7] M. Bianchi and A. Sagnotti, Phys. Lett. B247 (1990) 517

[8] G. Pradisi, A. Sagnotti and Y. S. Stanev, Phys. Lett. B354 (1995) 279; Phys. Lett. B356 (1995) 230

[9] A. Sagnotti and Y. S. Stanev, Fortsch. Phys. 44 (1996) 585 [Nucl. Phys. Proc. Suppl. 55B (1996) 200]

[10] B. Gato-Rivera and A.N. Schellekens, Nucl. Phys. B353 (1991) 519; Commun Math. Phys. 145, (1992) 85

[11] M. Kreuzer and A.N. Schellekens, Nucl. Phys. B411 (1994) 97

[12] J. Fuchs and Ch. Schweigert, Nucl. Phys. B558 (1999) 419; Nucl. Phys. B568 (2000) 543

[13] L.R. Huiszoon, A.N. Schellekens and N. Sousa, Phys. Lett. B470, (1999) 95; Nucl. Phys. B575 (2000) 401

L.R. Huiszoon and A.N. Schellekens, Nucl. Phys. B584 (2000) 705

L.R. Huiszoon, D-branes and O-planes in string theory - an algebraic approach, $\mathrm{Ph}$. D. thesis, Amsterdam 2002.

[14] J. Fuchs, L.R. Huiszoon, A.N. Schellekens, Ch. Schweigert and J. Walcher, Phys. Lett. B495 (2000) 427 